\title{
CSCW '92 Formal Video Program
}

\section{Saul Greenberg}

\author{
Department of Computer Science \\ University of Calgary \\ Calgary, Alberta \\ Canada T2N 1N4 \\ saul@cpsc.ucalgary.ca
}

Much of the experimental work in CSCW involves highly interactive systems and complex group interactions. While paper can convey the academic details of CSCW work, video is far more appropriate for capturing the true flavor and details of interactions. The CSCW conference has recognized the importance of video by creating its first refereed formal video program, published as a videotape in the SIGGRAPH Video Review series. Tapes can be purchased from:

\author{
SIGGRAPH Video Review Order Department \\ c/o First Priority \\ PO Box 576 \\ Itasca IL USA 60143-0576 \\ Phone: (within USA) 1-800-523-5503 \\ (outside USA) +1-708-250-0807 \\ Fax: $\quad+1-708-250-0038$
}

The sections below give a capsule summary of each video, followed by a list of references to related publications. As you will see, the formal video program is varied. Its contents cover enabling technologies, groupware prototypes, scenarios of real-life groupware use, and laboratory tools for analyzing group interaction.

\section{THE CONVERSATION BOARD}

Tom Brinck, Bellcore

The CONVERSATION BOARD is a prototype multi-user drawing application built to be used by people who are conversing over a distance, using a phone or a video phone system [2]. It was built in RENDEZVOUS TM, an experimental language and tool kit designed at Bellcore for constructing multi-user applications. The CONVERSATION BOARD is a structured graphics editor that provides "drawing" tools such as markers, lines, circles, rectangles, text, connectors, and images. Users can draw simultaneously and have telepointers, so their gestures are visible to each other. Images can be made "sticky", so that when a user draws onto an image, the drawing becomes grouped with the image. The video describes the first version of the CONVERSATION BOARD, and presents a scenario of one person giving directions to another by annotating a map.

Greenberg, S., ed. (1992). The CSCW '92 Formal Video Program. Proceedings of the ACM CSCW '92 Conference on Computer Supported Cooperative Work. Video in ACM SIGGRAPH Video Review (an optional supplement of Computer Graphics). Volume 87, ACM Press. Special Edition of the CSCW '92 Technical Video Program. Videotape.

\section{RUBBER ROCKS \\ Larry Koved, IBM}

The Veridical User Environments project is developing a Virtual Laboratory that brings together user interface technology, computational modeling of physical and nonphysical phenomena, and visualization. Virtual worlds technology provides human-computer interfaces to computational simulation and visualization systems. One challenge is in providing a means to allow people to collaborate on a computational model through its virtual worlds interface. The video demonstrates Rubber Rocks, a prototype multi-user virtual world that supports real-time conferencing. The application is a real-time simulation of objects modeled as point masses and springs. Through a hierarchically organized dialog manager, the single-user version of Rubber Rocks was easily transformed into a multi-user system [8].

\section{DELFT-WIT: RESEARCH ISSUES AND METHODS FOR BEHAVIORAL ANALYSIS \\ Jeroen van der Velden, Delft University}

Delft WIT-lab is a research environment for studying both human-computer interaction and CSCW [1]. The video describes a first series of experiments studying distributed group work, focusing on the experimental setup and the tools used to capture and analyze interaction data. In the experiment, small task teams played a management game under various interaction conditions for a period of ten weeks [9]. Data capture is through a computer-controlled video recorder that can record and play simultaneously on two or more tapes. A program developed at NPL-London allows video incidents to be indexed, retrieved, and edited. The video also shows a tool that registers the speech frequency and computer use of each group member during the experiment. With this tool, information can be derived of the group interaction - the participation of each group member, their speech duration, and the speech sequences.

\section{ENHANCED FACTORY COMMUNICATION Burke Magee and Glenna Cox, Boeing}

Capturing and conveying visual information is the most significant communication need of today's factory. 
Technologies now available on standard workstations can increase the quality and ease of visual communication within the factory and between the factory and companies around the world. With multimedia mail, text, photos, pictures, and motion video can be electronically mailed to remote locations. With real time information transmission, face to face meetings can be held between remotely located personnel within the factory. The video shows an example of how multimedia mail and computer-based conferencing can provide direct communication between a Boeing factory, its suppliers and its customers, thereby reducing communication flow time and the need for travel.

\section{COUPLING THE USER INTERFACES OF A MULTI-USER PROGRAM \\ Prasun Dewan, Purdue University}

An important question in the design, implementation, and use of multi-user programs is what should be the coupling between the user interfaces of a multi-user program? In other words, which objects should be shared among the users of a program and when should changes made by a user to a shared object be communicated to other users sharing it? The authors have developed a coupling model that allows users to control the coupling between their interfaces [3]. The model is based on a generalized multi-user editing interaction model, where multiple users view programs as active data that can be concurrently edited. The coupling model is implemented within SUITE, a framework that supports multi-user programs. The video uses a SUITE example to motivate and illustrate the main principles in the design of the coupling model.

\section{TOWARD SEAMLESS COLLABORATION MEDIA: FROM TEAMWORKSTATION TO CLEARBOARD} Hiroshii Ishii, Kazuho Arita and Minuro Kobayashi, NTT Human Interface Laboratories

This video presents the evolution of collaboration media design activities at NTT Human Interface Laboratories. "Seamlessness" has been a key concept of their evolving media design. The authors show the progression of their design from TEAMWORKSTATION to CLEARFACE to CLEARBOARD. TEAMWORKSTATION provides distributed users with a seamless shared workspace [6]. Each user can still use their favorite application programs or desktop tools, so there is only a minor cognitive seam between the individual and shared workspaces. CLEARBOARD permits coworkers in two different locations to draw with color markers or electronic pen while maintaining direct eye contact, gaze awareness, and the use of natural gestures [7].

\section{THE PANDORA MULTIMEDIA SYSTEM}

Andy Hopper, Olivetti

The PANDORA system makes available networked multimedia workstations to users in the Cambridge (UK) area [5]. The design is optimized for handling multiple audio and video streams with maximum flexibility. Many applications using both live and stored streams have been evaluated. Applications are integrated with an ACTIVE
BADGE system that provides location information of personnel. The system has been in use for two years and the most popular facilities are video-phone and video-mail.

\section{GROUPSKETCH}

Saul Greenberg and Ralph Bohnet

University of Calgary

GROUPSKETCH allows a small geographically-distributed group to list, draw, and gesture simultaneously in a communal work surface, supporting interactions similar to those occurring in the face-to-face drawing process [4]. It is a simple group sketching tool that allows its users to draw on a virtual piece of paper (the screen). GROUPSKETCH facilitates collaboration by: a) allowing gestural expression through large unique cursors visible on all displays; b) conveying the process of expressing ideas by transmitting small granular changes of user activity with minimum time delay; c) intermixing gestural, textual, and graphical expression modelessly; and d) providing simultaneous access to a common view of the work surface area, where any person can do any action at any time. The video illustrates several scenarios of GROUPSKETCH in action.

\section{REFERENCES}

1. Andriessen, J.H.T.H. and van der Velden, J.M. (1972) Back to process with synchoronous communication. Dept of Work and Organizational Psychology, Delft University, Delft, The Netherlands.

2. Brinck, T. and Gomez, L.M. (1992). A collaborative medium for the support of conversational props. In Proc Conderence on Computer-Supported Cooperative Work, Toronto, Canada, November 1-4, ACM Press.

3. Dewan, P. and Choudhary, R. (1991) Flexible user interace coupling in collaborative systems. In Proc CHI'91 Human Factors in Computing Systems, Monteray, CA, April 27-May 2, p41-49, ACM Press.

4. Greenberg, S. and Bohnet, R. (1991) "GroupSketch: A multi-user sketchpad for geographically-distributed small groups." In Proc Graphics Interface '91, Calgary, Alberta, June 5-7, p207-215.

5. Hopper, A. (1992) Improving Communications at the Desktop. In Communications after 2000AD: Report of the Royal Society Discussion Meeting, Chapman and Hall, March 18-19. In press.

6. Ishii, H. and Miyake, N. (1991) Toward an open shared workspace: Computer and video fusion approach of TeamWorkStation. Comm ACM 34:12, December.

7. Ishii, H., Kobayashi, M. and Grudin, J., (1992) Integration of inter-personal space and shared workspace: ClearBoard design and experiments. In Proc Conference on Computer-Supported Cooperative Work, Toronto, Canada, November $1-4$, ACM Press.

8. Koved, L., Codella, C., Jalili, R., Lewis, J.B., Ling, D.T., Lipscomb, J.S., Rabenhorst, D.A., and Wang, C.P. (1992) Dialog manager support For real-time conferencing. In Virtual Worlds, Research Report RC17968, IBM Research, P.O. Box 218, Yorktown Heights, NY 10598, April. 


\title{
ACM SI GGRAPH Video Review \#87
}

\author{
Saul Greenberg, Video Chair \\ Department of Computer Science, \\ University of Calgary \\ Calgary, Alberta, Canada T2N 1N4 \\ phone: +1 4032206087 \\ saul@cpsc.ucalgary.ca
}

Cite as:

Greenberg, S., ed. (1992). ACM SI GGRAPH Video Review (an optional supplement of Computer Graphics). Volume 87. Special Edition of the CSCW '92 Technical Video Program. Videotape, ACM Press.

A summary of this video is described in The CSCW '92 Formal Video Program. Proceedings of the ACM CSCW ' 92 Conference on Computer Supported Cooperative Work, 1992.

\section{Abstract and Contents}

Much of the experimental work in CSCW involves highly interactive systems and complex group interactions. While paper can convey the academic details of CSCW work, video is far more appropriate for capturing the true flavor and details of interactions. The CSCW conference has recognized the importance of video by creating its first refereed formal video program, published as a videotape in the SIGGRAPH Video Review series.

The CSCW'92 Formal Video Program description gives a capsule summary of each video, followed by a list of references to related publications.

1. The Pandora Multimedia System. Andy Hopper, Olivetti

2. Enhanced Factory Communications. Burke Magee and Glenna Cox, Boeing

3. Coupling the User I nterfaces of a Multi-User Program. Prasun Dewan, Purdue University

4. GroupSketch. Saul Greenberg and Ralph Bohnet University of Calgary

5. The Conversation Board. Tom Brinck, Bellcore

6. Toward Seamless Collaboration Media: From TeamWorkstation to ClearBoard. Hiroshii Ishii, Kazuho Arita and Minuro Kobayashi, NTT

7. Rubber Rocks. Larry Koved, IBM

8. Delft-WIT: Research I ssues and Methods for Behavioral Analysis. Jeroen van der Velden, Delft University 